\title{
Mikronährstoffe in der hausärztlichen Betreuung älterer Patienten
}

\author{
Stephan Wey
}

\section{Schlüsselwörter}

Geriatrie, Mikronährstoffmangel, orthomolekulare Therapie, Demenz, Morbus Parkinson

\section{Zusammenfassung}

Ältere Menschen sind aufgrund von Fehlernährung, Resorptionsstörungen, Erkrankungen und Medikamenteneinnahme gefährdet für Mikronährstoffmängel. Entsprechende Diagnostik und Supplementierung orthomolekularer Substanzen erscheint zur Prophylaxe chronischer und neurodegenerativer Erkrankungen sowie von Osteoporose, Sturzneigung und Infekten notwendig für ein „rüstiges“ Altern mit hoher Lebensqualität.

In der täglichen hausärztlichen Praxis gibt es eine Vielzahl geriatrischer Patienten, meist mit Polymorbidität und entsprechender Polymedikation. Die Versorgung dieser Patienten bindet in der schulmedizinischen Routine viel ärztliche Kompetenz und Zeit. Die Ernährung der älteren Generation wird meist durch kohlenhydratreiche Nahrungsmittel (Brot, Kartoffeln, Reis, Süßwaren), Milchprodukte (Milch, Käse, Quark, Joghurt), Wurstwaren und akzeptable Mengen Obst und/oder Gemüse geprägt. Hochwertiges, mastfreies Fleisch, Fisch, Nüsse, Eier oder Pilze werden seltener konsumiert, was zu einem Mangel der gerade in diesen Lebensmitteln enthaltenen orthomolekularen Stoffe (siehe Diagnostik) führen kann. Daraus resultieren eine Vielzahl der Befindlichkeitsstörungen und Krankheiten bei geriatrischen Patienten und ein unnötig schwerer oder irrever-

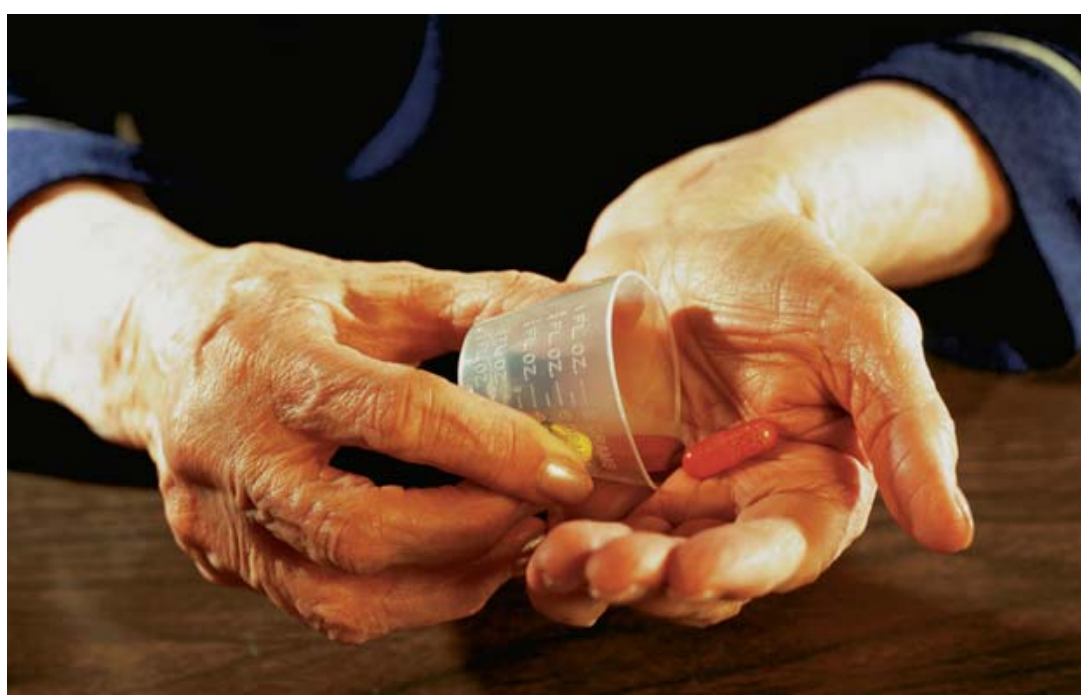

Besonders bei polymorbiden älteren Menschen, die zahlreiche Medikamente einnehmen, besteht ein erhöhtes Risiko für Mikronährstoffmängel. @ Dynamic Graphics

sibler Verlauf, z.B. bei diversen neurodegenerativen Erkrankungen.

\section{Diagnostik}

Die in der hausärztlichen Routine kontrollierten Laborwerte beinhalten selten mehr als das kleine Blutbild, Leber- und Nierenwerte, Blutzucker, Cholesterin und Triglyzeride, die Elektrolyte Natrium, Kalium und Kalzium sowie TSH basal. Die Erfassung einer Organfunktion sagt jedoch kaum etwas über die Pathophysiologie und Biochemie der Zellen aus und die o. g Werte verändern sich nur bei entsprechend starker Schädigung der Organe. Viele - oft unspezifische - Symptome, die von Patienten geklagt werden, wie Müdigkeit, sind auch nicht in der Bildgebung anderer diagnostischer Verfahren wie Sonografie, EKG, Lungenfunktion oder radiologi- schen Verfahren zu ergründen. Die Aussage, „dann sei das eben das Alter“, kann vor dem Hintergrund leicht behandelbarer Mikronährstoffdefizite so nicht hingenommen werden und jeder Arzt ist hier aufgerufen, erweiterte orthomolekulare Diagnostik zu betreiben.

Als Basis für eine optimale Zellfunktion sind viele orthomolekulare Stoffe lebenswichtig. Aus der Praxiserfahrung fehlen geriatrischen Patienten neben Eiweiß und Aminosäuren (L-Carnitin u.a.) hauptsächlich $\omega$-3-Fettsäuren, Magnesium, Selen, Zink, Vitamin $\mathrm{B}_{12}$ und $\mathrm{D}_{3}$ sowie Coenzym Q10, gelegentlich auch Vi$\operatorname{tamin} \mathrm{B}_{6}$. Eine ergänzende Messung dieser Werte hilft in der Einschätzung der geklagten Beschwerden bzw. der vorhandenen Multimorbidität deutlich weiter. Dabei ist zu beachten, dass die genaue Einschätzung des Mangels nicht alleine auf Serummessungen beruhen darf. Der überwiegend zelluläre Gehalt 
von Magnesium, Selen und Zink wird optimaler über eine Vollblutmessung ermittelt, Coenzym Q10 über die cholesterinkorrigierte Messung und Vitamin $\mathrm{B}_{12}$ über Parameter wie Holotranscobalamin (Holo-TC), Homocystein im Plasma oder auch Methylmalonsäure im Morgenurin oder Serum. In die spätere therapeutische Entscheidung müssen klinische Parameter und Symptome einfließen.

\section{Orthomolekulare Substanzen}

\section{Natrium und Kalium}

Im Gegensatz zum meist ausreichend über die Nahrung aufgenommenen Kalzium gibt es im Alter viele Störfaktoren für die Elektrolyte Natrium und Kalium. Natrium kann durch die meist salzarme Ernährung der Älteren fehlen und wird zusätzlich durch Diuretika ausgeschieden. Zudem gibt es eine Vielzahl von Möglichkeiten einer sekundären Überproduktion von Antidiuretischem Hormon mit daraus folgender Hyponatriämie, z.B. zerebrale Erkrankungen (Subarachniodalblutung, Hirnatrophie, Zentralvenenthrombose), Medikamente (Carbamazepin, Neuroleptika, Antidepressiva), Paraneoplasie, Sarkoidose, Stress, Schmerzen, Übelkeit, akute Psychose oder Hypothyreose. Bei dauerhaft $<115 \mathrm{mmol} / \mathrm{l}$ und akut $<125 \mathrm{mmol} / \mathrm{l} \mathrm{lie}-$ genden Serumwerten drohen

- Hirnödem mit Somnolenz,

घerwirrtheit,

- Muskelkrämpfe und

- Koma.

Neben der Therapie der Ursache können Empfehlungen zu salzreicherer Ernährung und ggf. Kochsalztabletten gegeben werden.

Kalium ist im Zusammenspiel mit Natrium essenziell für zahlreiche zelluläre Funktionen, speziell Muskel-, Nieren- und Nerventätigkeit. Eine Hypokaliämie kann eine Vielzahl von Ursachen haben. Neben mangelnder Zufuhr über die Nahrung liegt es meist an übermäßigem Kaliumverlust, der oft mit erhöhtem Wasserverlust, z.B. als Folge von Erbrechen und Durchfall, einhergeht. Im Rahmen eines Conn-Syndroms kann es durch gesteigerte renale Kaliumausscheidung zu einer Hypokaliämie kommen. Bei Älteren sind die häufig eingesetzten Diuretika und $\mathrm{Ab}$ führmittel von Bedeutung. Bei Hypokaliämie können auftreten:

- Adynamie
Kopfschmerzen
Verstopfung
Schwäche bis Lähmungen der Extre-
mitätenmuskulatur
arrhythmogene Wirkung von Digita-
lispräparaten und Katecholaminen
ventrikuläre und supraventrikuläre
Extrasystolen bis hin zu Vorhof- oder
Kammerflimmern

Neben der Ursachenbekämpfung sind Diuretika bei älteren Menschen kritisch einzusetzen, auch vor dem Hintergrund des Ausschwemmens anderer Mineralien und Spurenelemente wie Natrium, Magnesium und Zink sowie den potenziell negativen Auswirkungen auf Glukose- und Harnsäurestoffwechsel.

Eine kaliumreiche Ernährung besteht aus Früchten, Kartoffeln, Avocados, Spinat, Tomaten und Gemüsesäften, Fleisch, Milch, Bohnen und Pilzen. Bei Supplementation ist eine orale $\mathrm{Zu}$ fuhr von Kalium ausreichend, entweder als Monopräparat oder in Kombination mit Magnesium.

\section{Magnesium}

Magnesium ist Cofaktor von Enzymen und Reaktionen, an denen ATP beteiligt ist. Es dient der Membranstabilisierung, ist ein natürlicher Kalziumantagonist und aktiviert die Acetylcholin-Freisetzung aus Synapsen. Bei Abfall der extrazellulären Magnesiumkonzentration kommt es zu einem Anstieg der intrazellulären Kalziumkonzentration, was sich zytotoxisch und lipidperoxidierend auswirkt. Die Folgen sind

- erhöhte Katecholaminausschüttung mit Stressintoleranz oder Ängsten,

neuromuskuläre Übererregbarkeit mit Muskelverspannung bis hin zu -krämpfen,
- Schwindel,

Kopfschmerzen,

- Extrasystolie und

Bronchialobstruktion.

Grüne Pflanzen, Getreide, Gemüse und Nüsse sind Hauptlieferanten von Magnesium. Jedoch sind die überdüngten Böden zunehmend ausgelaugt und magnesiumarm. Neben Mangelernährung tragen aber auch chronische Niereninsuffizienz, Diabetes mellitus, chronisch entzündliche Darmerkrankungen, Alkoholkonsum und Diuretika zum Magnesiummangel bei. Aktive Magnesiumeinnahme von ca. $370 \mathrm{mg} / \mathrm{d}$ wirkt blutdrucksenkend [8].

Durch die überwiegend intrazelluläre Magnesiumverteilung (wie Zink und Selen) ist ein klinisch relevanter Mangel schon bei niedrignormalen Serumwerten $(<0,8 \mathrm{mmol} / \mathrm{l}) \quad \mathrm{zu}$ erwarten. Eine Vollblutmessung gibt den tatsächlichen zellulären Magnesiumgehalt genauer wieder. Bei der oralen Ergänzung ist zu beachten, dass nur 30\% der Dosis resorbiert werden und die Verträglichkeit durch die Diarrhöneigung limitiert ist.

Übliche Dosis einer Substitution ist 300$600 \mathrm{mg} / \mathrm{d}$.

\section{Zink}

Dieses essenzielle Spurenelement ist Bestandteil von weit über 200 Enzymen. Zink erfüllt wichtige Funktionen im Insulinstoffwechsel. Bei einem Mangel drohen verminderte Insulinsekretion, gestörte Insulinfreigabe und dadurch stark schwankende Blutzuckerwerte sowie eine gestörte Glukoseutilisation. Das Spurenelement ist ein Baustein der intrazellulären Synthese von Nukleinsäuren und Proteinen und dient der Verbesserung der humoralen und zellulären Immunität. Zink ist als Coenzym für die Decarboxylierung von 5-Hydroxytryptophan zu Serotonin obligat.

Immunologische Folgen eines Mangels sind Verschlechterungen des zellulären und humoralen Immunstatus: schlechtere Phagozytose, Komplementaktivierung, Antikörperproduktion, 
Lymphopenie, reduzierte Aktivität der Natürlichen Killerzellen (NK), Verschiebung hin zu den „katabolen“ IL-1 $\beta$, IL-6, TNF- $\alpha$, Cortisol und Adrenalin.

Es gibt zahlreiche Störfaktoren, die zu einem niedrigen Zinkspiegel führen können. In höherem Lebensalter betrifft dies besonders Verluste über Stuhl, Urin und Schweiß, aber auch Nikotinabusus, Medikamenteneinnahme (Zinkkomplexe durch Cortison, Chelatbildner, Tetracycline, verschiedene Diuretika, ACEHemmer; Hemmung der Zinkresorption durch Phosphat, Kalzium, Alkohol, Laxantienabusus, Phenytoin; außerdem durch Antacida, Zytostatika, Antidepressiva, Antirheumatika), mangelnde Zufuhr bei Reduktionsdiäten und Vegetariern. Typische Mangelsymptome sind v ständige Müdigkeit, Erschöpfung,

- schlechte Wundheilung und Dekubitusneigung,

- trockene/entzündliche schuppige Haut,

— rezidivierende Erkältungen,

Infektanfälligkeit,

- brüchige Haare und Nägel,

- Gewichtsabnahme, Myopathie,

- gestörter Geschmacks- und Geruchssinn, Appetitlosigkeit,

verzögerte Rekonvaleszenz,

- Depression, Stimmungslabilität, Gereiztheit,

- Apathie, Hirnleistungsstörungen.

Ältere Menschen mit einer Substitution von $20 \mathrm{mg}$ Zink/d für 2 Jahre hatten signifikant weniger Infekte der Atemwege (ähnlich bei $100 \mu$ g Selen/d) [4]. Serumwerte $<800 \mu \mathrm{g} / \mathrm{l}$ sollten an einen klinisch relevanten Zinkmangel denken lassen. Zinkhaltige Nahrungsmittel sind insbesondere Fleisch, Meeresfrüchte, Fisch und Nüsse, sowie Getreide und Hülsenfrüchte, diese allerdings bei deutlich eingeschränkter biologischer Verwertbarkeit wegen der anhängenden Phytinsäure.

Die tägliche Substitution liegt bei $10 \mathrm{mg} / \mathrm{d}$ dauerhaft und bis $50 \mathrm{mg} / \mathrm{d}$ bei akutem Mangel oder Erkrankungen.

\section{Selen}

Selen ist ein essenzielles Spurenelement mit hoher antientzündlicher und immunmodulierender Potenz und ist damit in der Immunologie des Älteren mit Häufung von Krebs und (chronisch) entzündlichen Erkrankungen nicht wegzudenken. Die Konversion von fT4 zu fT3 ist selenabhängig, sodass eine unzureichende Versorgung mit Selen zu klinischer Hypothyreose führen kann. Fleisch, Fisch, Meeresfrüchte, Paranüsse und Pistazien, Getreide, Eigelb und auch Rosenkohl, Zwiebeln und Knoblauch sind selenhaltig. Aufgrund mangelnder Aufnahme mit der Nahrung besonders bei den selenarmen Böden Südwestdeutschlands kann das Spurenelement ebenso fehlen wie bei Malabsorptionssyndromen (Morbus Crohn, Zöliakie), Diabetikern, Rauchern, erhöhter Schwermetallbelastung, Niereninsuffizienz oder Dialysepflichtigkeit und Veganern.

Die übliche Substitution beträgt initial $300 \mu \mathrm{g}$ idealerweise als Natriumselenit, bei akuten Erkrankungen bis $900 \mu \mathrm{g} / \mathrm{d}$. In der Dauereinnahme reichen oft $100 \mu \mathrm{g} / \mathrm{d}$ aus. Nach stabilisierten Wirkspiegeln bei mind. 100-140 $\mu \mathrm{g} / \mathrm{l}$ können als Dauertherapie durchaus die günstigeren Selenhefeprodukte verwendet werden mit Tagesdosierungen von höchstens $50-80 \mu \mathrm{g}$, da diese stärker in den endogenen Pool eingebaut werden.

\section{Vitamin $\mathbf{B}_{6}$}

Das wasserlösliche Vitamin wirkt als Coenzym in etwa 100 enzymatischen Reaktionen überwiegend im Aminosäurestoffwechsel mit. Im Nervensystem gehört es zu den Cofaktoren der Neurotransmitterbildung. Bei der Zelldifferenzierung weisen Studien auf eine geringere Krebsinzidenz bei normalen Blutspiegeln hin.

Vitamin $\mathrm{B}_{6}$ kommt in geringen Dosen in fast allen Lebensmitteln tierischer und pflanzlicher Herkunft vor. Milchprodukte, Leber, Geflügel und Fleisch, Fisch, Kohl, grüne Bohnen, Linsen, Feldsalat, Kartoffeln, Vollkorngetreide und -produkte, Weizenkeime, Nüsse, Hefe, Weißbier,
Avocados und Bananen sind gute Quellen.

An einen Mangel muss man denken bei

- Appetitverlust,

Zungenbrennen,

Dermatitis,

mikrozytär-hypochromer Anämie,

Ataxie,

unklaren Krämpfen und

Angststörungen.

Ein optimaler Wirkspiegel ist bei $>25 \mu \mathrm{g} / \mathrm{l}$ im EDTA-Blut zu sehen.

Bei Mangel füllen $100 \mathrm{mg}$ p.o./d rasch die Speicher auf. Ohne Dauertherapie fallen diese jedoch oft bald wieder ab, sodass eine niedrigdosierte regelmäBige Einnahme $(10-20 \mathrm{mg})$ besonders bei neurologischen Erkrankungen sowie erhöhtem Homocystein oder Kryptopyrrol sinnvoll ist.

\section{Vitamin $\mathrm{B}_{12}$}

Hefe deckt praktisch den gesamten BKomplex ab und schon 1 EL z.B. zum Binden von Soßen oder Würzen könnte den Tagesbedarf weitgehend abdecken. Für eine unzureichende Versorgung prädestinieren neben einer fleischarmen Mangelernährung eine Vielzahl von Vitamin- $\mathrm{B}_{12}$-Resorptionsstörungen im Gastrointestinaltrakt, die bei Älteren vorkommen können (chronisch atrophe Gastritis, Pankreasatrophie, Pankreasinsuffzienz, chronisch entzündliche Darmerkrankungen, säurehemmende Medikamente, Metformin, Antibiotika, Antikonvulsiva). Bei Leber- und Nierenerkrankungen ist eine erhöhte Vitamin$\mathrm{B}_{12}$-Ausscheidung möglich.

Bei geriatrischen Patienten müssen

- Gangataxie,

distal betonte Parästhesien,

- Gang- und Standunsicherheit,

- „Manschettengefühl“ um die Fußknöchel herum,

- brennende Zunge,

- makrozytäre Anämie,

घ unklare LDH- und Bilirubinerhöhung sowie

- neuropsychiatrische Symptome wie Depression, Konzentrationsstörungen und Gedächtnisstörungen 
an einen Vitamin- $B_{12}$-Mangel denken lassen [7]. Bei Demenzpatienten finden sich bei bis zu 70\% der Patienten niedrige $\mathrm{B}_{12}$-Spiegel [12]. Bei längerer Dauer des Morbus Parkinson und einer L-Dopa-Therapie entsteht ein Vitamin- $B_{12}-$ Mangel mit vermehrtem Auftreten von Neuropathien [14].

Bei Unklarheiten ist neben der o.g. Diagnostik auch die probatorische Gabe von mindestens 8-10 Injektionen $1000 \mu \mathrm{g}$ Vitamin $B_{12}$ i.m. sinnvoll, um die Vitamin- $B_{12}$-Speicher sicher aufzufüllen. Bei klinischem Erfolg dieser Maßnahme ist auf Dauer auf einen hochnormalen Wirkspiegel (mind. $>450 \mu \mathrm{g} / \mathrm{l}) \mathrm{zu}$ achten, der mit ca. 4-6wöchentlichen Injektionen meist gehalten werden kann.

\section{$\alpha$-Liponsäure}

Dieses hauptsächlich in Fleisch vorkommende Vitaminoid ist neben $\mathrm{B}-\mathrm{Vi}$ taminen mitochondrial am zellulären Stoffwechsel und der ATP-Produktion beteiligt. Beim Typ-2-Diabetiker ist eine Verbesserung von peripherer Insulinsensitivität, zellulärer Glukoseaufnahme und -oxidation bekannt. Über direkte oder enzymatische Wirkung ist $\alpha$-Liponsäure proenergetisch, antioxidativ und antiinflammatorisch wirksam und kann offensichtlich neuronale Schäden durch „advanced glycation endproducts" (AGE) verhindern bzw. abmildern [5]. Neben der bekannten Indikation Polyneuropathie ist daher der Einsatz zur Verhinderung oder Progressionsminderung der Demenz und anderer neurodegenerativer Erkrankungen wie Morbus Parkinson sinnvoll. Die ersten Studien sind vielversprechend [11].

\section{Die übliche Dosis beträgt $600 \mathrm{mg} / \mathrm{d}$.}

\section{Vitamin $\mathrm{D}_{\mathbf{3}}$}

Es gibt eine Vielzahl an neuen Erkenntnissen zu Vitamin $\mathrm{D}_{3}$. Neben der altbekannten Indikation Osteoporose sind Studiendaten zu praktisch allen akuten und chronischen inflammatorischen Erkrankungen [3], Krebs [9], arterieller Hypertonie [2], Diabetes [13], chroni- schen Schmerzen [18] und vielen neurodegenerativen Erkrankungen wie kognitive Einschränkungen [1] und Demenz [10] positiv. Bei Morbus Parkinson werden ebenfalls häufig niedrigere Vitamin- $\mathrm{D}_{3}$-Werte gemessen [20] und der Schweregrad sowie die Sturzneigung der Parkinsonpatienten nehmen bei niedrigen Werten zu [21]. Die Vitamin$\mathrm{D}_{3}$-Spiegel (25-OH) im Serum der Älteren sind z.T. dramatisch niedrig und dies betrifft im Gegensatz zu den aktuellen Empfehlungen zur Vitamin- $\mathrm{D}_{3}$ Gabe nicht nur Pflegebedürftige, sondern fast alle Älteren [16]. Offensichtlich wurde die Alterung von Haut und synthesepflichtigen Organen (Leber, Nieren) sowie der Sonnenschutz (Kleidung, Hüte, Creme) der Senioren bisher unterschätzt. Die wenigen wirklich Vitamin$\mathrm{D}_{3}$-reichen Nahrungsmittel wie fette Fische, Eier („vom Kardiologen verboten“) oder Avocados stehen nicht häufig auf dem Speiseplan. In meiner Praxis habe ich bei Laborkontrollen kaum einen Senior mit normalen Vitamin- $\mathrm{D}_{3}$-Spiegeln erlebt. Das betrifft auch die aktiven Senioren, die auf dem Lande bis ins hohe Alter regelmäßig ihre Gärten pflegen.

Daher ist Senioren durchweg zu empfehlen, mindestens 1000 I. E. Vitamin $D_{3}$ täglich zuzuführen und an allen Tagen ohne Sonnenlichtexposition am Abend weitere 1000-2000 I.E. einzunehmen, besonders konsequent in den Herbst-| Wintermonaten.

Damit sind optimale Wirkspiegel von $>75 \mathrm{nmol} / \mathrm{l}$ meist erreichbar. Ältere Menschen ernähren sich selten kalziumarm und leiden eher unter Obstipation, die durch Kalziumeinnahme noch verstärkt wird. Daher erwarte ich mittelfristig, dass die Fachgesellschaften die ergänzende Kalziumempfehlung bei Osteoporose zurücknehmen. Der Kalziumhaushalt wird bekanntlich durch Vitamin $D_{3}$ wesentlich gesteuert mit höherer enteraler Kalziumaufnahme, verminderten renalen Kalziumverlusten und aktivem ossären Kalziumeinbau.

\section{Coenzym Q10}

Es ist ein essenzieller Bestandteil der Atmungskette der Mitochondrien und dort an der Bildung von ATP, also der Energiegewinnung der menschlichen Zelle, beteiligt. Als fettlösliches Antioxidans hemmt Coenzym Q10 die Lipidperoxidation.

Tierische Lebensmittel (Innereien, Fleisch, Fisch, Eier), Hülsenfrüchte und Biosynthese in der Leber aus Vorstufen sind die wesentlichen Quellen für Coenzym Q10. Daher haben Vegetarier, Veganer und chronisch Leberkranke mit einem Mangel zu rechnen. Ein häufiges Problem stellt die Verschreibung von Statinen dar, die durch die HMG-CoAReduktasehemmung in der Leber nicht nur cholesterinsenkend sind, sondern auch die Biosynthese von Coenzym Q10 blockieren. In Studien mit Statinpatienten sinkt die Myopathiehäufigkeit signifikant bei Zugabe von 100-200 mg Coenzym Q10/d. Weitere positive Daten liegen u.a. für Herzmuskelerkrankungen und eine Substitution bei Morbus Parkinson [17] vor. Eine neuere Arbeit bestätigte einen gehäuft bei Parkinsonpatienten vorkommenden Mangel an Coenzym Q10 [22]. Der Normalspiegel von $>0,67 \mathrm{mg} / \mathrm{l}$ im EDTA-Blut $(>0,20$ cholesterinkorrigiert) sollte je nach Grunderkrankung therapeutisch bis $>3 \mathrm{mg} / \mathrm{l}$ gesteigert werden. Das reduzierte Ubiquinon ist biologisch wirksamer und hat möglicherweise Vorteile. Die erhältlichen Präparate schwanken in Qualität und Preis jedoch stark, weswegen Spiegelkontrollen unbedingt notwendig sind.

Die Dosierung reicht von $30 \mathrm{mg}$ als prophylaktische Nahrungsergänzung bis hin zu therapeutischen Dosierungen meist zwischen 100 und 300 mg/d.

\section{w-3-Fettsäuren}

Diese Fettsäuren mit den Hauptvertretern Eicosapentaensäure (EPA) und der neuroaktivierenden Docosahexaensäure (DHA) kommen in der modernen industriellen Ernährung und bei dem hohen Konsum an Produkten aus $\omega-6-$ 
reichen Masttieren prozentual in immer geringerem Maß vor. Bei regelmäßigen Laborkontrollen findet sich meist nur ein $\omega$-3-Index von $3-5 \%$ und durchschnittlich niedrigere Werte bei Vegetariern, die das Fehlen der tierischen Quellen mit Konsum pflanzlicher Quellen (Linolensäure) meistens nicht ausgleichen können. Ein optimaler Index läge bei über $8 \%$. Eine Vielzahl von Wirkungen auf Endothel und den zellulären Stoffwechsel (antiinflammatorische Kaskade, vasodilatierend, lipidregulierend) und der klare Mangel in der geriatrischen Ernährung sollten daher dringend zu einer ergänzenden Therapie führen.

Durch die Framingham-Studie und weiteren Untersuchungen ist bei hohen DHA-Spiegeln ein bis zu $47 \%$ selteneres Auftreten von Demenz publiziert [15]. Es gibt positive Daten zu Hirnleistung, Krebs, Immunschwäche, Depression [6], Schizophrenie, chronischer Bronchitis, Osteoporose und MS. Aktuelle Erkenntnisse auch bei Älteren sind unter [23] zusammengefasst.

Die Dosis liegt bei mind. 500-1000 mg EPA und DHA/d in möglichst reinen Präparaten.

\section{L-Carnitin}

Es wird endogen aus den essenziellen Aminosäuren Methionin und Lysin in Nieren, Leber und auch Gehirn gebildet, wobei die Vitamine $\mathrm{B}_{3}, \mathrm{~B}_{6}$ und $\mathrm{C}$ sowie Eisen die Cofaktoren sind. Wichtigste Quelle ist Fleisch, sodass sich bei Älteren mit geringerem Fleischkonsum, Vegetariern oder gar Veganern rasch Mängel einstellen können. Erhöhte Ausscheidung findet man bei Diabetikern, Dialysepflichtigen, Langzeitmedikation mit Valproat oder Krebspatienten unter Chemotherapie mit Cisplatin oder Ifosfamid.

L-Carnitin fördert die Bereitstellung von Energie in der Zelle besonders in peripheren Muskeln und im Myokard, aktiviert T-Lymphozyten sowie NK-Zellen und ist mitochondrialer Schutz gegen freie Radikale. Ein Mangel wirkt sich daher klinisch als
- myokardiale Pumpminderung,

veriphere Muskelschwäche,

a allgemeine Asthenie,

घ Immunschwäche und möglicherweise als

* Beschleuniger einer Alzheimerdemenz

aus. Durch nicht mitochondrial metabolisierte Fettsäuren kann ein L-Carnitinmangel über den Einbau in Cholesterin oder Triglyzeride zu einer Plaquebildung und verstärkter Arteriosklerose beitragen.

Eine optimale Diagnostik umfasst neben dem Gesamtcarnitin das freie Carnitin im Serum und die Carnitinester. Ein Mangel wird durch eine optimierte Zufuhr von Muskelfleisch und eine therapeutische Gabe von L-Carnitin erreicht.

Neben Kapseln und Trinklösungen bieten sich bei akuten Beschwerden und Mangelnachweis Infusionen an. Die Tagesdosis beträgt dabei ca. $4 \mathrm{~g}$.

\section{Besonderheit Homocystein}

Homocystein entsteht im Stoffwechsel aus der Aminosäure Methionin. Zum Rückumbau werden die Vitamine $\mathrm{B}_{6}$, $B_{12}$ und Folsäure sowie Cholin (aus Lecitihin) benötigt. Anhand der vorherigen Ausführungen sind gerade aufgrund möglicher Mängel von Vitamin $\mathrm{B}_{6}$ und $B_{12}$, aber auch Cholin bei Älteren und Rauchern, bei Kachexie und Mangelernährung (sowie bes. bei alkoholkranken Senioren auch Folsäure) Erhöhungen des Homocysteins zu erwarten. Dies gilt ebenso für Typ-2-Diabetiker, Patienten mit chronischer Niereninsuffizienz und bei Medikamenteneinnahme (MTX, Carbamazepin, Phenytoin).

Durch Oxidation von Cholesterin und Lipoproteinen unter dem Einfluss von Homocystein kommt es im Endothel zur Bildung von Schaumzellen als Vorläufer arteriosklerotischer Plaques. Zusätzlich hat Homocystein eine thrombogene Wirkung über eine Steigerung der Fibrinogensynthese und Bindung von
Lipoprotein(a) an Fibrin und erhöht freie Radikale. Die Neurotoxizität erklärt sich durch einen N-Methyl-D-Aspartat-Agonismus.

Das kardiovaskuläre Risiko und die Demenzhäufigkeit steigen mit der Höhe des Homocysteinspiegels an [19]. Homocystein ist ein guter Biomarker einer unzureichenden Stoffwechselsituation z. B. des Diabetikers und/oder der 3 o.g. Vitamine. Stoffwechseloptimierung und eine optimale ergänzende Therapie mit Vitamin $\mathrm{B}_{6}, \mathrm{~B}_{12}$ und evtl. auch Folsäure müssen bedacht werden.

Ein Beispiel für die erfolgreiche orthomolekulare Therapie eines multimorbiden älteren Patienten lesen Sie in der Kasuistik ab S. 26.

Interessenkonflikte: Der Autor erklärt, dass keine wirtschaftlichen oder persönlichen Verbindungen bestehen.

\section{Literatur}

[1] Annweiler C, Fantino B, Schott AM et al. Vitamin D insufficiency and mild cognitive impairment: cross-sectional association. Eur J Neurol 2012; 19: 1023-1029

[2] Burgaz A, Orsini N, Larsson SC, Wolk A. Blood-25-hydroxyvitamin D concentration and hypertension: a meta-analysis. J Hypertens 2011; 29: 636-645

[3] Ginde AA, Mansbach JM, Camargo CA. Association between serum-25-hydroxyvitamin $D$ level and upper respiratory tract infection in the Third National Health and Nutrition Examination Survey. Arch Intern Med 2009; 169: 384-390

[4] Girodon F, Galan P, Monget AL et al. Impact of trace elements and vitamin supplementation on immunity and infections in institutionalized elderly patients. Min. Vit. Aox. geriatric network. Arch Int Med 1999; 159: 748-754

[5] Gröber U. Neuroenhancement mit $\alpha$-Liponsäure. ZKM 2012; 1: 45-49

[6] Jazayeri S, Tehrani-Doost M, Keshavarz SA et al. Comparison of therapeutic effects of omega-3 fatty acid eicosapentaenoic acid and fluoxetine, separately and in combination, in major depressive disorder. Austr New Zeal J Psychiatry 2008; 42: 192-198 
[7] Jesse S, Ludolph AC. Die Vitamine $B_{1}, B_{6}$ und $B_{12}$. Der Nervenarzt 2012; 83: $521-534$

[8] Kass L, Weekes ], Carpenter L. Effect of magnesium supplementation on blood pressure: a meta-analysis. Eur J Clin Nutr 2012; 66: 411-418

[9] Lappe JM, Travers-Gustafson D, Davies KM et al. Vitamin D and calcium supplementation reduces cancer risk: results of a randomized trial. Am J Clin Nutr 2007; 85: 1586-1591

[10] Llewellyn DJ, Langa K, Lang I. Serum 25-hydroxyvitamin D concentration and cognitive impairment. J Geriatr Psychiatry Neurol 2009; 22: 199-195

[11] Maczureck A, Hager K, Kenklies M et al. Lipoic acid as an anti-inflammatory and neuroprotective treatment for Alzheimer's disease. Adv Drug Deliv Rev 2008; 60: 1463-1470

[12] Nourhashemi F, Gillette-Guyonnet S, Andrieu S. Alzheimer disease: protective factors. Am J Clin Nutr 2000; 71: 643S-649S

[13] Parkera J, Hashmia O, Dutton D. Levels of vitamin D and cardiometabolic disorders: systematic review and meta-analysis. J Maturitas 2009; 65: 225-236

[14] Rajabally YA, Martey J. Neuropathy in Parkinson disease: prevalence and determinants. Neurology 2011; 77: 1947-1950

[15] Schaefer EJ, Bongard V, Beiser AS et al. Plasma phosphatidylcholine docoshexaenoic acid content and risk of dementia and Alzheimer disease: the Framingahm Heart Study. Arch Neurol 2006; 63: 1545-1550

[16] Schilling S. Epidemic vitamin D deficiency among patients in an elderly care rehabilitation facility. Dtsch Arztebl Int 2012; 109: 33-38

[17] Shults CW, Oakes D, Kieburtz K. Effects of coenzyme Q10 in early Parkinson disease: evidence of slowing of the functional decline. Arch Neurol 2002; 59: 1541-1550

[18] Turner MK, Hooten WM, Schmidt JE et al. Pain Med 2008; 9: 979-984

[19] Van Dam F, Van Gool WA. Hyperhomocysteinemia and Alzheimer's disease: A systematic review. Arch Gerontol Geriatr 2009; 48: 425-430

[20] Evatt ML, DeLong, MR, Khazai N et al. Prevalence of Vitamin D insufficiency in patients with Parkinson disease and Alzheimer disease. Arch Neurol 2008; 65: 1348-1352

[21] Ding $H$, Dhima K, Lockhart KC et al. Unrecognized vitamin $D_{3}$ deficiency is common in Parkinson disease. Neurology 2013; 81: 1531-1537

[22] Mischley L, Allen J, Bradley R. Coenzyme Q 10 deficiency in patients with Parkinsons's disease. J Neurol Sci 2012; 318: 72-75

[23] von Schacky C. Hirnstruktur und Hirnfunktion: Die Rolle der Omega-3Fettsäuren. Zs f Orthomol Med 2014; 1: 20-23

online: http://dx.doi.org/10.1055/s-0034-1368498

$\begin{aligned} & \text { Dr. med. Stephan Wey } \\ & \text { Facharzt für Innere Medizin } \\ & \text { Naturheilverfahren, Palliativmedizin, Notfallmedizin } \\ & \text { Laufbachstr. } 38 \\ & 77886 \text { Lauf } \\ & \text { E-Mail: s.wey@wey-partner.de }\end{aligned}$
Schwerpunkten Ernährung, orthomolekulare Medizin, komplementäre
Onkologie und Hyperthermie niedergelassen. Regelmäßige Vortrags-
tätigkeit, Vorstandsmitglied der Ärztegesellschaft für Erfahrungsheil-
kunde e.V. und Deutschen Gesellschaft für Hyperthermie e. V.

